\title{
THE ASYMPTOTIC BEHAVIOR OF A SOLUTION OF THE THIRD ORDER LINEAR DIFFERENTIAL EQUATION
}

\author{
J. D. SCHUUR
}

We shall consider the differential equation

$$
x^{\prime \prime \prime}+a(t) x^{\prime \prime}+b(t) x^{\prime}+c(t) x=0,
$$

where $x$ is real and $a(t), b(t)$, and $c(t)$ are real valued and continuous for $0 \leqq t<\infty$, and we shall prove:

THEOREM. If $\Lambda(r, t)=r^{3}+a(t) r^{2}+b(t) r+c(t)=0$ is the characteristic equation of (1) and if the characteristic roots are real and satisfy $\lambda_{1}(t)$ $<\alpha<\lambda_{2}(t)<\beta<\lambda_{3}(t)$ for all $t \geqq 0$, where $\alpha$ and $\beta$ are constants, then (1) has a solution of the form $x(t)=x(0) \exp \int_{0}^{t} \xi(s) d s$ where $\alpha<\xi(t)<\beta$ for $0 \leqq t<\infty$.

C. Olech [2] proved a similar theorem for the second order equation. As an alternate means of proof, he suggested the topological method of T. Ważewski and that approach is used here. The details of Ważewski's method may be found in [3] or [1, pp. 179-182].

Proof of THE THEOREM. Write (1) as the system

$$
x^{\prime}=y, \quad y^{\prime}=z, \quad z^{\prime}=-c(t) x-b(t) y-a(t) z .
$$

We seek a solution $(x(t), y(t), z(t))$ of (2) such that $x(t)>0$ and $\alpha<y(t) / x(t)<\beta$ for $0 \leqq t<\infty$, or $(x(t), y(t), z(t), t) \in T=\{(x, y, z, t)$ : $x>0, \alpha x<y<\beta x,-\infty<z<\infty, 0 \leqq t<\infty\}$ for all $t \geqq 0$.

We will use the theorem of Ważewski: if all points of egress of $T$ are points of strict egress and if there exists a set $Z \subset T \cup S$ ( $S$ is the set of egress) such that $Z \cap S$ is a retract of $S$ but not of $Z$, then there is at least one point $P=\left(x_{0}, y_{0}, z_{0}, t_{0}\right) \in Z$ such that if $(x(t), y(t), z(t))$ is the solution of (2) with $\left(x\left(t_{0}\right), y\left(t_{0}\right), z\left(t_{0}\right), t_{0}\right)=P$, then $(x(t)$, $y(t), z(t), t) \in T$ for all $t \geqq t_{0}$.

Now the boundary of $T$ is $F=A \cup B \cup C$ where

$$
\begin{aligned}
& A=\{(x, y, z, t): x>0, y=\beta x,-\infty<z<\infty, 0 \leqq t<\infty\}, \\
& B=\{(x, y, z, t): x>0, y=\alpha x,-\infty<z<\infty, 0 \leqq t<\infty\}, \text { and } \\
& C=\{(x, y, z, t): x=y=0,-\infty<z<\infty, 0 \leqq t<\infty\} .
\end{aligned}
$$

Let $(x(t), y(t), z(t))$ be a solution of (2) such that $(x(\tau), y(\tau), z(\tau), \tau) \in A$. Then $(y-\beta x)(\tau)=0$ and $(y-\beta x)^{\prime}(\tau)=\left(z-\beta^{2} x\right)(\tau)$. If $z<\beta^{2} x$, we have

Received by the editors May 31, 1966. 
no points of egress; if $z>\beta^{2} x$, we have points of strict egress. If $\left(z-\beta^{2} x\right)(\tau)=0$, consider $\left(z-\beta^{2} x\right)^{\prime}(\tau)=\left(-c x-b y-a z-\beta^{2} y\right)(\tau)$ $=-x(\tau)\left(c+b \beta+a \beta^{2}+\beta^{3}\right)(\tau)=-x(\tau) \Lambda(\beta, \tau)>0$. Then $(y-\beta x)(t)>0$ for $|t-\tau|$ small and $(x(\tau), y(\tau), z(\tau), \tau)$ is not a point of egress. (The graph of $\Lambda(r, t)$ versus $r$, for $t$ fixed, shows that $\Lambda(\beta, t)<0$. If we assume $\gamma<\lambda_{1}(t)<\delta \leqq \alpha<\lambda_{2}(t)<\beta<\lambda_{3}(t)$ for all $t \geqq 0$, or similar bounds for $\lambda_{3}(t)$, and repeat the preceding argument using $\gamma$ and $\delta$ in place of $\alpha$ and $\beta$, we find that in the last step $\left(z-\delta^{2} x\right)^{\prime}(\tau)=-x(\tau) \Lambda(\delta, \tau)<0$ and we have points of weak egress.)

Let $A^{*}=\left\{(x, y, z, t): x>0, y=\beta x, z>\beta^{2} x, 0 \leqq t<\infty\right\}$; these are the points of strict egress in $A$.

By similar reasoning we find that on $B$ : if $z<\alpha^{2} x$, we have points of strict egress; and if $z \geqq \alpha^{2} x$, we have no points of egress. Let $B^{*}=\left\{(x, y, z, t): x>0, y=a x, z<\alpha^{2} x, 0 \leqq t<\infty\right\}$.

Now let $(x(t), y(t), z(t))$ be a solution of (2) such that $(x(\tau), y(\tau), z(\tau), \tau) \in C$. By uniqueness, $\{x=y=z=0,0 \leqq t<\infty\}$ contains no points of egress. Looking at the trace of any other such solution in the $x, y$-plane, $d y / d x=y^{\prime}(t) / x^{\prime}(t)=z(t) / y(t) \rightarrow \infty$ or $-\infty$ as $t \rightarrow \tau$. In either case, the solution is tangent to the $y, z$-plane as $t \rightarrow \tau$ and hence neither enters nor leaves $T$. Thus, $C$ contains no points of egress.

Therefore, $S$ (the set of egress points) equals $S^{*}$ (the set of strict egress points) and $S^{*}$ has two components, $A^{*}$ and $B^{*}$. Let $Q=\left(x_{1}, y_{1}, z_{1}, 0\right) \in A^{*}, R=\left(x_{2}, y_{2}, z_{2}, 0\right) \in B^{*}$, and $Z=\sigma Q+(1-\sigma) R$, $0 \leqq \sigma \leqq 1$. Then $Z \subset T \cup S$ and $Z \cap S=\{Q, R\}$ is a retract of $S$, but not of $Z$. Thus, there exists a $P \in Z$ such that if $(x(t), y(t), z(t))$ is the solution of $(2)$ with $(x(0), y(0), z(0), 0)=P$, then $(x(t), y(t), z(t), t)$ $\in T$ for all $t \geqq 0$.

If, in addition to the hypotheses of the theorem, $\gamma<\lambda_{i}(t)<\delta$ ( $i=1$ or 3 ) for $0 \leqq t<\infty$ and $\gamma^{2}<\delta^{2}$, then we may consider the region

$$
U=\left\{(x, y, z, t): x>0, \gamma x<y<\delta x, \gamma^{2} x<z<\delta^{2} x, 0 \leqq t<\infty\right\}
$$

and by similar arguments, using further assumptions (for example: $b(t)<-\delta^{2} ;$ or $-\delta^{2}<b(t)<-\gamma^{2}$ and $\gamma \delta^{2}+\delta^{2} a(t)+\gamma b(t)+c(t)>0$; or $-\gamma^{2}<b(t)$ and $\gamma^{2} \delta+\gamma^{2} a(t)+\delta b(t)+c(t)<0<\gamma \delta^{2}+\delta^{2} a(t)+\gamma b(t)$ $+c(t)$; for all $t \geqq 0)$, we may show that the boundary of $U$ contains no points of egress and hence every solution starting in $U$ remains in $U$ for all $t \geqq 0$. But these conditions seem unnatural.

Perhaps the other (in the sense of linear independence) two solutions have different behavior. In particular, we have shown the existence of one nonoscillatory solution, but the other two solutions might oscillate. 
ADDED IN PROOF. The following was suggested by L. K. Jackson, but he proved it differently. If we take a second point $R_{1} \in B^{*}$ and repeat the last paragraph of the proof using $Q$ and $R_{1}$ in place of $Q$ and $R$, we may obtain a second solution of (1) which also satisfies the conclusion of the theorem. And if $R_{1} \neq k R, k$ a real number, these two solutions will be linearly independent.

\section{REFERENCES}

1. L. Cesari, Asymptotic behavior and stability problems in ordınary differential equations, Springer-Verlag, Berlin, 1959.

2. C. Olech, Asymptotic behavior of solutions of second order differential equations, Bull. Acad. Polon. Sci. 7 (1959), 319-326.

3. $\mathrm{T}$. Ważewski, Une méthode topologique de l'examen du phenomène asymptotique relativement aux équations différentielles ordinaires, Rend. Accad. Lincei (8) 3 (1947), 210-215.

Michigan State University 